2011-11-21

\title{
Bonding of Polydimethylsiloxane Microfluidics to Silicon-Based Sensors
}

Long-Fang Tsai

I2112ben@yahoo.com.tw

William C. Dahlquist

Seunghyun Kim

Gregory P. Nordin

nordin@byu.edu

Follow this and additional works at: https://scholarsarchive.byu.edu/facpub

Part of the Electrical and Computer Engineering Commons

\section{Original Publication Citation}

Long-Fang Tsai, William c. Dahlquist, Seunghyun Kim and Gregory P. Nordin, "Bonding of Polydimethylsiloxane Microfluidics to Silicon-Based Sensors", J. Micro/Nanolith. MEMS MOEMS 1, 439 (211), DOI: 1.1117/1.3659139

\section{BYU ScholarsArchive Citation}

Tsai, Long-Fang; Dahlquist, William C.; Kim, Seunghyun; and Nordin, Gregory P., "Bonding of Polydimethylsiloxane Microfluidics to Silicon-Based Sensors" (2011). Faculty Publications. 78. https://scholarsarchive.byu.edu/facpub/78 accepted for inclusion in Faculty Publications by an authorized administrator of BYU ScholarsArchive. For more information, please contact ellen_amatangelo@byu.edu. 


\section{Micro/Nanolithography, \\ MEMS, and MOEMS}

\section{Bonding of polydimethylsiloxane microfluidics to silicon-based sensors}

Long-Fang Tsai

William C. Dahlquist

Seunghyun Kim

Gregory P. Nordin 


\section{Bonding of polydimethylsiloxane microfluidics to silicon-based sensors}

\author{
Long-Fang Tsai \\ William C. Dahlquist \\ Seunghyun Kim \\ Gregory P. Nordin \\ Brigham Young University \\ Electrical and Computer Engineering \\ Provo, Utah 84602 \\ E-mail: nordin @byu.edu
}

\begin{abstract}
We investigate bonding polydimethylsiloxane (PDMS) to silicon using a thin $(\sim 2 \mu \mathrm{m})$ intermediate adhesive layer stamped onto a PDMS piece prior to bonding. In particular, we compare as adhesive layers Sylgard 184 and 182 curing agents and a UV curable adhesive (NOA 75). We examine the effect of both curing temperature and duration on curing agent bond strength. Bond strengths for the different adhesives are determined by measuring the average burst pressure at a PDMS-silicon interface using a PDMS test design. We find that Sylgard 184 curing agent gives the highest bond strength with burst pressure of $700 \mathrm{kPa}$ or more for curing at either $60^{\circ} \mathrm{C}$ for $3 \mathrm{~h}, 70^{\circ} \mathrm{C}$ for $30 \mathrm{~min}$, or $90^{\circ} \mathrm{C}$ for $20 \mathrm{~min}$. Curing at room temperature takes substantially more time with an average burst pressure of 433 and $555 \mathrm{kPa}$ for curing times of 16 and $27 \mathrm{~h}$, respectively. In comparison, Sylgard 182 curing agent takes $32 \mathrm{~h}$ at room temperature to achieve a burst pressure of $289 \mathrm{kPa}$, while NOA 75 with a $50^{\circ} \mathrm{C} 12 \mathrm{~h}$ post-UV exposure bake yields a burst pressure of $125 \mathrm{kPa}$. (C) 2011 Society of Photo-Optical Instrumentation Engineers (SPIE). [DOI: 10.1117/1.3659139]
\end{abstract}

Subject terms: polydimethylsiloxane; bonding; silicon; microfluidics.

Paper 11001RR received Jan. 3, 2011; revised manuscript received Aug. 25, 2011; accepted for publication Oct. 17, 2011; published online Nov. 21, 2011.

\section{Introduction}

Over the last several decades much attention has been focused on development of reliable and rapid approaches to detect desired target analytes in fluids for applications that include medical diagnostics, proteomics, and drug screening. A limiting factor for such biosensors can be the potentially long response time required for detection of low concentration (sub-picomolar) target analytes. It has been shown that, depending on the primary mass transport method of the target as well as the sensor's physical dimensions and transduction efficiency, it may take anywhere from hours to days before sufficient transport of molecules to the sensing surface occurs. ${ }^{1}$ It is widely recognized that the response time can be reduced through development of microfluidic devices in order to rely not only on diffusion but also convection to contribute toward rapid analyte transport. ${ }^{2}$

As an example, we are developing silicon microcantilever arrays with a simultaneous in-plane photonic transduction technique as a label-free, multiplexed sensing platform. ${ }^{3-6}$ In our approach, each microcantilever is functionalized with a receptor layer prior to integration of a microfluidic cover chip on top of a silicon microcantilever chip. Hence, the integration process must preserve the efficacy and affinity of already-deposited receptor molecules on each microcantilever. This imposes severe constraints on the integration process thermal budget, particularly in the case of proteinbased receptor molecules. Note that this is in contrast to more typical configurations in which channel surfaces are functionalized after the device has been assembled.

At present, we attach two-layer poly(dimethylsiloxane) $(\mathrm{PDMS})^{7,8}$ microfluidics with integrated valves, fluid chan-

$1932-5150 / 2011 / \$ 25.00$ @ 2011 SPIE nels, and control channels directly onto silicon-on-insulator (SOI) microcantilever chips. PDMS has several characteristics that render it attractive for use in prototyping microfluidic structures including its optical transparency, elastomeric nature, and permeability to air. Unfortunately, as a result of a relatively low shear modulus (typically between $100 \mathrm{kPa}$ and $3 \mathrm{MPa}$ ), the bond between PDMS and other surfaces cannot withstand high amounts of pressure without the aid of a bond enhancement technique. ${ }^{9}$ Several methods exist for reversibly or irreversibly bonding PDMS to silicon. A common procedure involves surface treatment techniques such as plasma oxidation to enhance bond strength. ${ }^{10-12}$ However, such surface treatments remain stable in air for only a short time (a few minutes in our experience), creating a strict time constraint to align and attach a PDMS part to a substrate once the PDMS part is removed from the plasma etching tool. ${ }^{9}$

Use of an intermediate layer is an alternate approach to facilitate bonding between two materials. ${ }^{12,13}$ For instance, ultraviolet (UV) curable adhesive has been used as an intermediate layer between substrates, such as glass and silicon as reported in Ref. 13. PDMS-to-silicon bonding was also demonstrated in Ref. 13, but at a cure temperature of $90^{\circ} \mathrm{C}$, which is incompatible with our process in which microcantilever functionalization occurs before microfluidic integration. Reference 12 reports use of a relatively thick layer ( $\sim 150 \mu \mathrm{m}$, i.e., $\sim 2.5$ times thicker than the height of fluid channels used in our microfluidics) of adhesion promoter on a variety of materials, including silicon, to facilitate bonding with PDMS that is applied in an uncured state. The PDMS is then cured at $80^{\circ} \mathrm{C}$, which, again, is incompatible with our process.

In this paper we present a new method for bonding PDMS to silicon using curing agent as an intermediate adhesive layer, which is one of the two components that are mixed to 


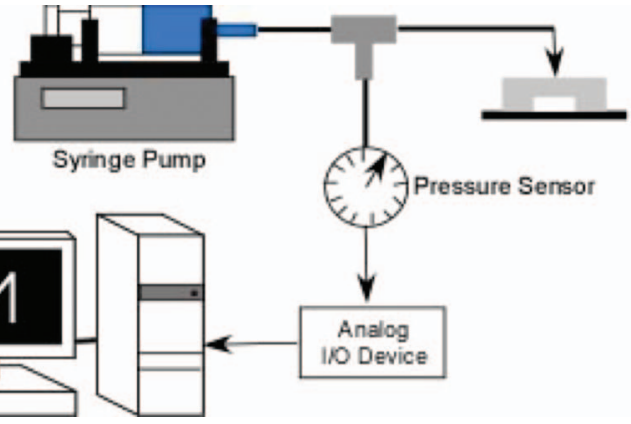

Fig. 1 Experimental setup.

form PDMS. Specifically, we investigate both Sylgard 184 and 182, each of which is bonded to silicon with its corresponding curing agent. We explore a range of curing times and temperatures, including room temperature bonding, to facilitate comparison of relative bond strengths for different treatments. In addition, we examine a UV curable epoxy [Norland Optical Adhesive (NOA 75)] as an alternate adhesive layer. In all cases, bond strength is evaluated by measuring burst pressure when simple PDMS-silicon test samples with a single chamber are subjected to increasing internal pressure. ${ }^{13}$ Finally, a similar test is performed using a much more complex microfluidic PDMS device integrated onto an actual silicon-on-insulator microcantilever chip to qualitatively evaluate the pressure such devices need to withstand in practice.

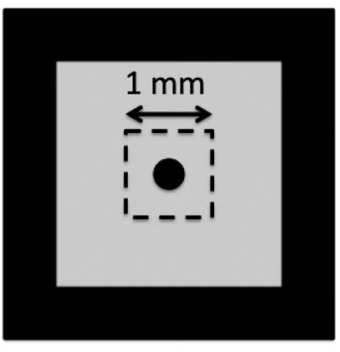

(a)

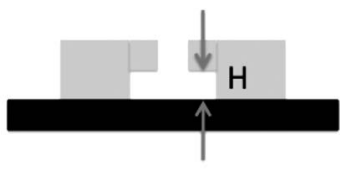

Silicon (b)

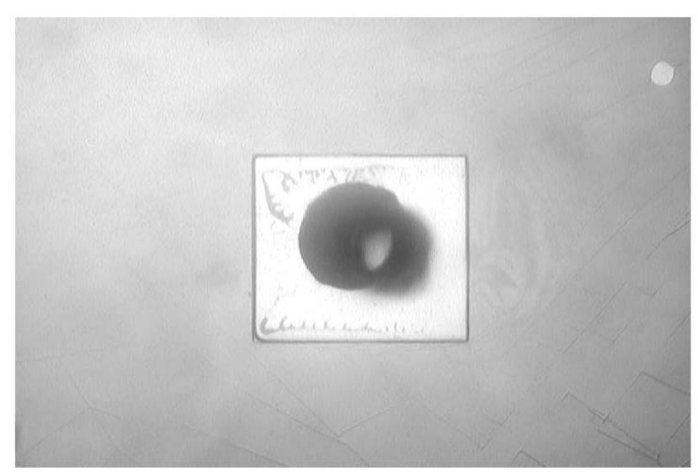

(c)

Fig. 2 (a) Top view and (b) side view schematic diagrams of an assembled test device with $H_{i}=40 \mu \mathrm{m}$. (c) Microscope image of inner $1 \mathrm{~mm}$ square of test device showing $0.5-\mathrm{mm}$ diameter hole.

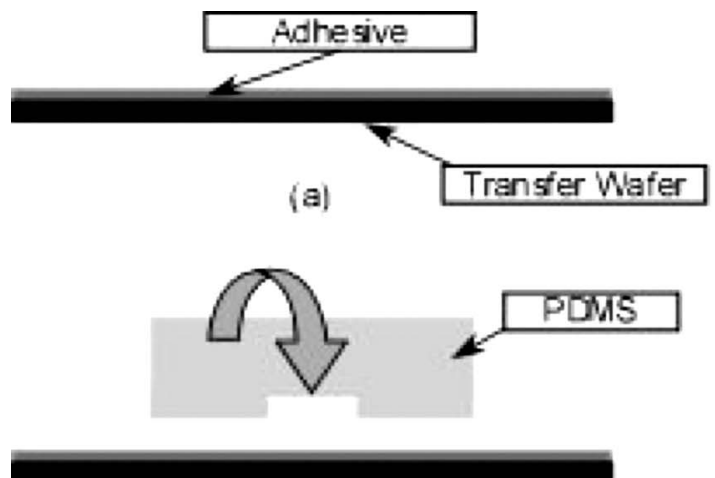

(b)

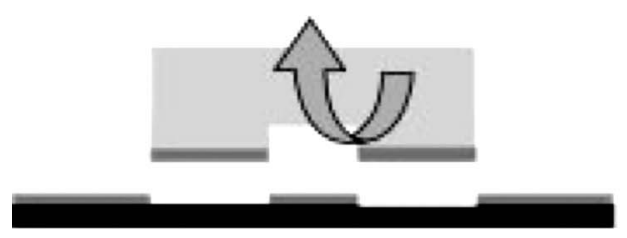

(c)

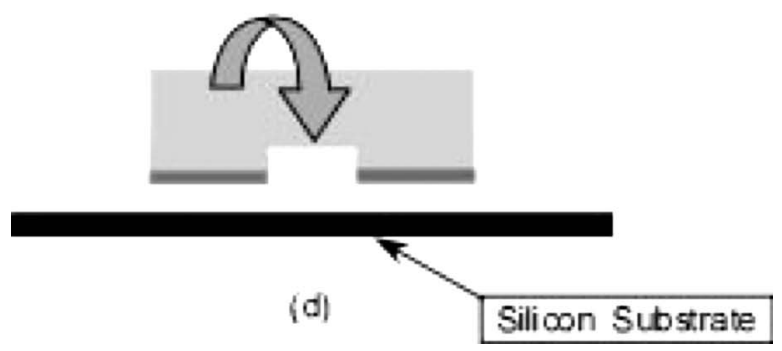

Fig. 3 Assembly procedure for device construction. (a) Transfer wafer is spin coated with thin layer of adhesive. (b) PDMS sample is placed in contact with adhesive. (c) PDMS is lifted off removing adhesive with it. (d) Adhesive-coated PDMS is placed onto a silicon-based device.

\section{Experimental}

\subsection{Burst Pressure Test}

Several methods exist for testing the bond strength between materials, such as load bearing mechanisms or razor-blade tests. ${ }^{12,14}$ For many applications it is beneficial to know at what pressure delamination and eventual leaking of sample fluid may occur between the PDMS and silicon substrate. For this reason, we chose to apply a burst pressure measurement as reported in Ref. 13 to test the reliability of the bond using an intermediate adhesive layer. Figure 1 illustrates our experimental setup for measuring burst pressure, which we define as the pressure at which delamination occurs at the PDMS-silicon interface.

A measurement is made by injecting fluid [deionized (DI) water] with a syringe pump into an inlet-only PDMS/adhesive/Si device and forcing entrapped air out of the fluid chamber through the PDMS, which is gaspermeable. Once all air is removed, the syringe pump is used to ramp the pressure in the device, which is recorded by a microgauge pressure sensor (Honeywell, model 24PCFFA6G). Pressure as a function of time is acquired with a National 
Instrument Analog I/O device using custom LABVIEW data acquisition software.

\subsection{Test Device Fabrication}

Using standard techniques ${ }^{9}$ based on patterning photoresist on a blank silicon wafer, a fluid mold is created and used to fabricate PDMS devices with 10:1 mixing of a PDMS base to the curing agent. For both Sylgard 184 and 182 the PDMS is cured at $80^{\circ} \mathrm{C}$ for $60 \mathrm{~min}$. Before being separated from the mold, a $0.5-\mathrm{mm}$ diameter hole is punched through the center of the inner square of each PDMS device to create a fluid inlet port. Figures 2(a) and 2(b) show top and side view schematic diagrams of an assembled test device. A microscope image of the inner square with inlet port is shown in Fig. 2(c). The inner square is designed to be $1 \mathrm{~mm} \times 1 \mathrm{~mm}$ with a height, $H_{i}$, of $40 \mu \mathrm{m}$ while the outer square is $10 \mathrm{~mm} \times 10 \mathrm{~mm}$ with a height, $H_{o}$, of $\sim 2.3 \mathrm{~mm}$.

\subsection{Test Device Assembly}

We implement a stamping method to apply adhesive to the PDMS surface. ${ }^{13}$ The same method is used for both UV epoxy and curing agent; however, preassembly sample preparation is slightly more involved for the case of UV epoxy, as explained below. For each test the adhesive is spin coated onto a transfer wafer at $4000 \mathrm{rpm}$ for $1 \mathrm{~min}$. With the curing agents and UV epoxy having a viscosity of close to $100 \mathrm{cP}$, a similar film thickness is produced at the end of spin coating $(2.13 \mu \mathrm{m}$ for Sylgard 184 and $2.43 \mu \mathrm{m}$ for NOA $75)$. For curing agent tests, the PDMS is stamped onto the transfer wafer and removed, taking with it the thin adhesive layer. When applying UV epoxy, because of the hydrophobic nature of PDMS and in order to obtain a uniform layer on the surface, the PDMS requires a brief $(1 \mathrm{~min})$ oxygen plasma treatment to make the surface more hydrophilic. Otherwise, after the PDMS is removed from the transfer wafer the epoxy forms into beads on the surface thereby resulting in a nonuniform layer. Note that this brief treatment is easily implemented prior to stamping with epoxy, particularly since no alignment step is needed as would be the case when bonding PDMS to silicon with only a plasma treatment.

The next step is to gently, with minimal shear, place the coated PDMS piece onto a silicon wafer. The assembly procedure is illustrated in Fig. 3. For the case of a curing agent, a variety of curing temperatures and durations are investigated. For curing temperatures above room temperature, samples are placed on a hotplate. For each combination of curing time and temperature five samples are prepared and tested. In the case of UV epoxy, it is cured according to manufacturer recommendations using a mask aligner (Karl Suss MA150) as a UV light source with a 30 min exposure for a dose of $16 \mathrm{~J} / \mathrm{cm}^{2}$. A steel pin with attached tubing is inserted into the hole in the PDMS to connect the syringe pump to the device.

\section{Results and Discussion}

Average burst pressure is determined as shown in Fig. 4 for the example of Sylgard 184 PDMS bonded with curing agent at $70 \mathrm{C}$ for $30 \mathrm{~min}$. The pressure for each of the five samples

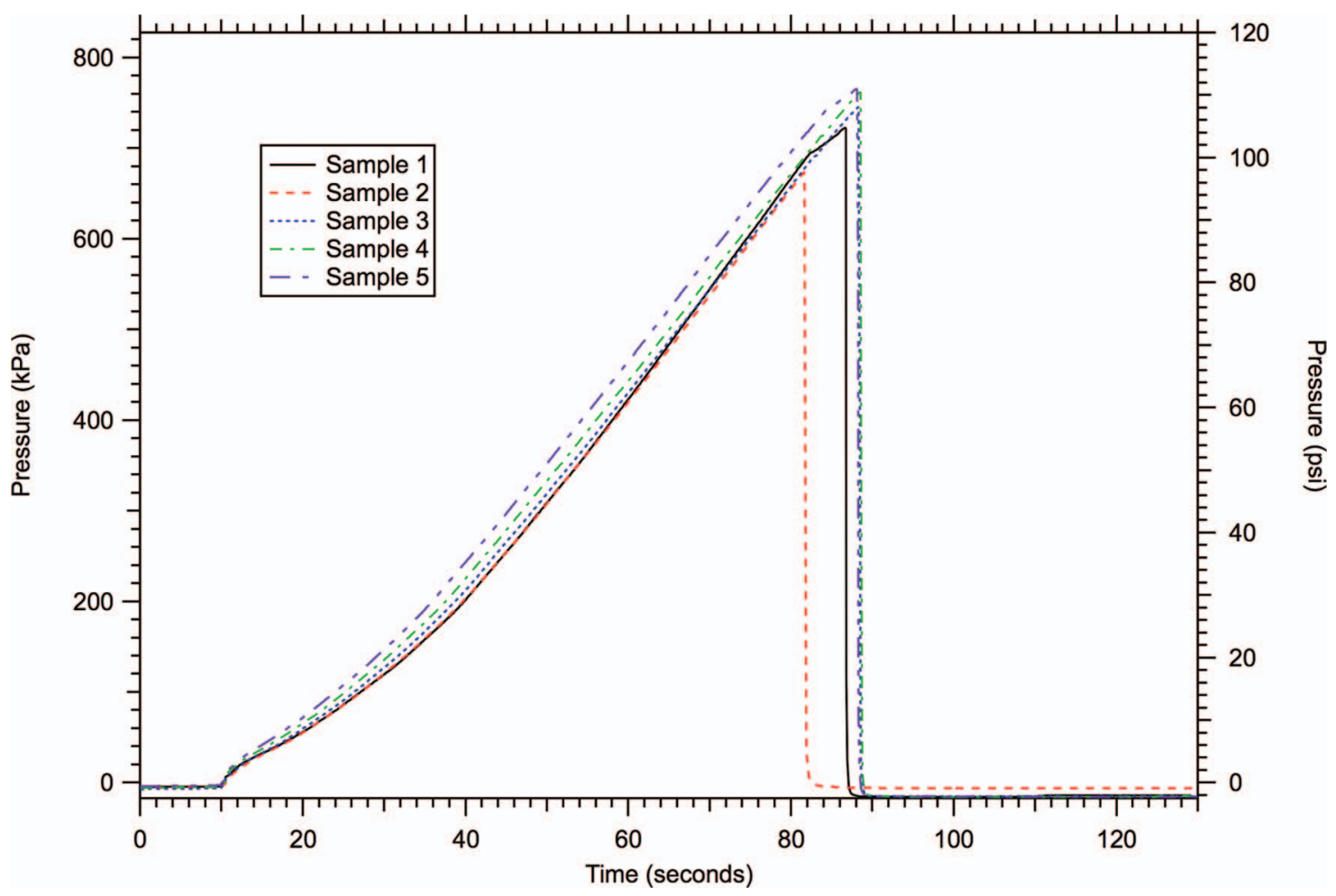

Fig. 4 Pressure at the test device as a function of time for five Sylgard 184 samples with cured curing agent as adhesive bonded at $70^{\circ} \mathrm{C}$ for $30 \mathrm{~min}$. 
Tsai et al.: Bonding of polydimethylsiloxane microfluidics to silicon-based sensors

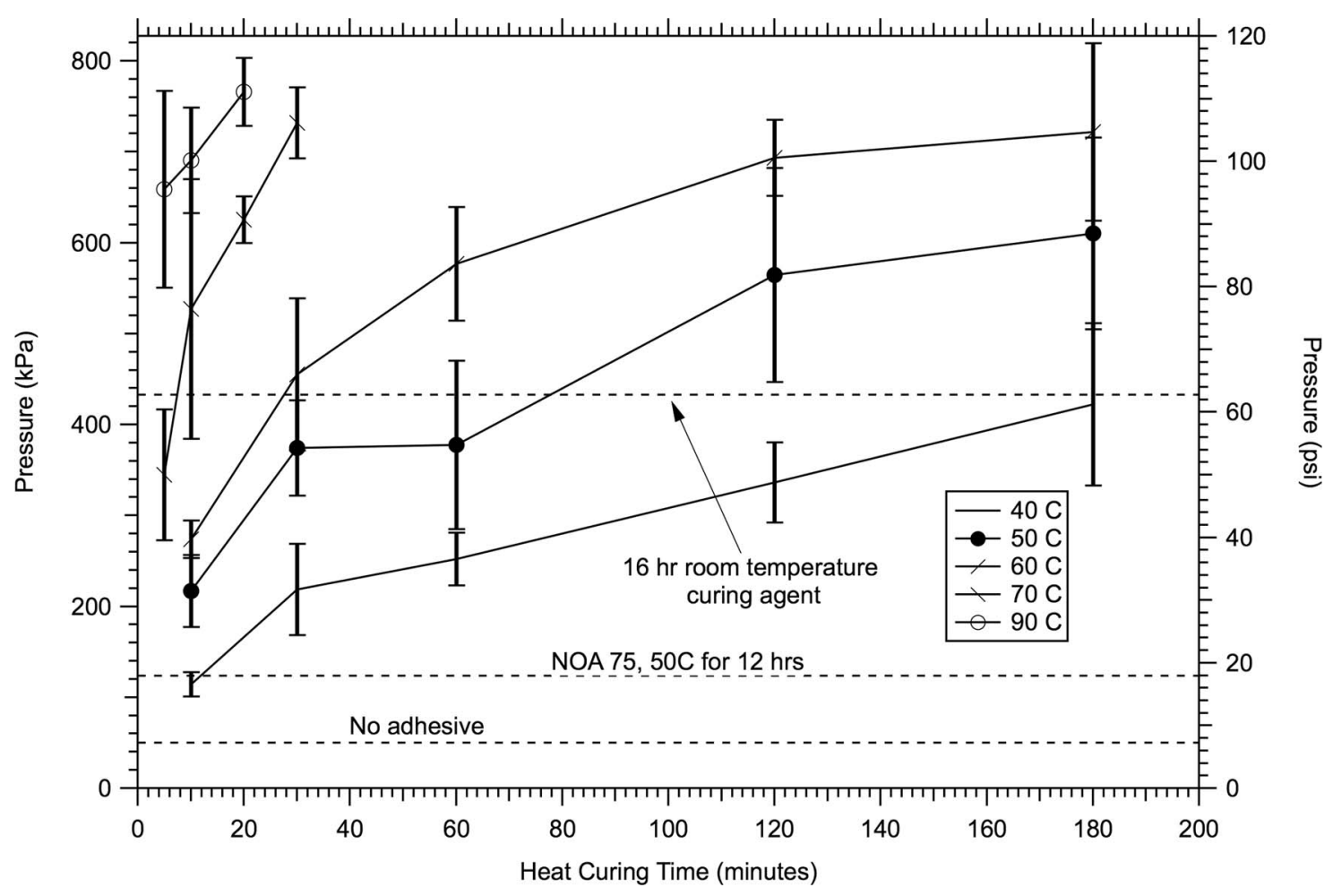

Fig. 5 Average measured burst pressure as a function of curing time for different curing temperatures for Sylgard 184. Error bars indicate \pm one standard deviation.

is shown as a function of time as the syringe pump is used to increase pressure in the sample. The graph shows increasing pressure until the PDMS delaminates from the silicon, at which point the pressure drops drastically. The maximum pressure is the burst pressure for that sample.

Figure 5 shows the measured average burst pressure for a variety of bonding temperatures and times for Sylgard 184 PDMS with Sylgard 184 curing agent as the adhesive. To provide a baseline for comparison, five PDMS dies were bonded at room temperature with no intermediate adhesive to silicon. Only the natural tackiness of PDMS is used to create the bond. In this case the average burst pressure is $50 \mathrm{kPa}$. With curing agent as the adhesive and a $90^{\circ} \mathrm{C}$ curing temperature, a burst pressure of $659 \mathrm{kPa}$ is achieved after only $10 \mathrm{~min}$ cure time, and $766 \mathrm{kPa}$ after $20 \mathrm{~min}$. Burst pressures above $\sim 800 \mathrm{kPa}$ were not measureable because an air leak at the PDMS/pin interface would develop first, regardless of whether the steel pin was simply inserted into the PDMS or glued with epoxy, superglue, or uncured PDMS that was subsequently cured. Note in Fig. 5 that for $70^{\circ} \mathrm{C}$ curing temperature the burst pressure is $731 \mathrm{kPa}$ after $30 \mathrm{~min}$, but it takes a curing time of $3 \mathrm{~h}$ at $60^{\circ} \mathrm{C}$ to reach a comparable burst pressure.

UV curable epoxy exhibits a burst pressure of only $38 \mathrm{kPa}$ with just UV cure. As indicated in Fig. 5, the burst pressure increases to $124 \mathrm{kPa}$ when samples are baked at $50^{\circ} \mathrm{C}$ for $12 \mathrm{~h}$ after UV exposure. This is nearly a factor of 5 less than the burst pressure for curing agent at $50^{\circ} \mathrm{C}$ for $3 \mathrm{~h}(610 \mathrm{kPa})$. Curing agent is clearly superior in terms of bond strength to NOA 75 as a PDMS-to-silicon bonding agent.

As shown in Fig. 6, we performed the same measurements (i.e., same curing times and temperatures) for Sylgard
182 PDMS with Sylgard 182 curing agent as for Sylgard 184 in Fig. 5. Note that in all cases the average burst pressures are significantly lower than those obtained for Sylgard 184, indicating that Sylgard 184 is a better choice to form higher strength bonds for a given curing temperature and time. This is likely related to the fact that Sylgard 184 is formulated by the manufacturer to yield a faster cure than 182, which may indicate the formation of a denser crosslinked bond network in the silcon polymer with a concomitant increase in material strength.

We also examined room temperature curing of Sylgard 184 and 182 as shown in Fig. 7. Much longer curing times were required to obtain high bond strengths. For example, for Sylgard 184 curing for $16 \mathrm{~h}$ at room temperature yields a burst pressure $(433 \mathrm{kPa})$ comparable to curing at $70^{\circ} \mathrm{C}$ for $30 \mathrm{~min}$ (see Fig. 5). Curing for $27 \mathrm{~h}$ increases the burst pressure to $555 \mathrm{kPa}$. Sylgard 182 again shows substantially lower burst pressures, achieving $289 \mathrm{kPa}$ after $32 \mathrm{~h}$.

Since our microcantilever sensor preparation process involves aminosilane deposition before PDMS bonding, we also measured burst pressure of Sylgard 184 bonded to silicon samples on which a monolayer of 3-aminopropyldiisopropylethoxysilane (APDIES) was deposited overnight in anhydrous toluene. The burst pressure after $24 \mathrm{~h}$ is $281 \mathrm{kPa}$, which is approximately half of what it would have been for bonding to bare silicon.

All of the above results were obtained with PDMS fabricated the day before being bonded. We also investigated the effect of letting PDMS sit in covered Petri dishes for a number of days prior to bonding. As shown in Fig. 8, we observed no degradation in burst pressure for PDMS used within 7 days of PDMS fabrication. Additional measurements indicate that 
Tsai et al.: Bonding of polydimethylsiloxane microfluidics to silicon-based sensors

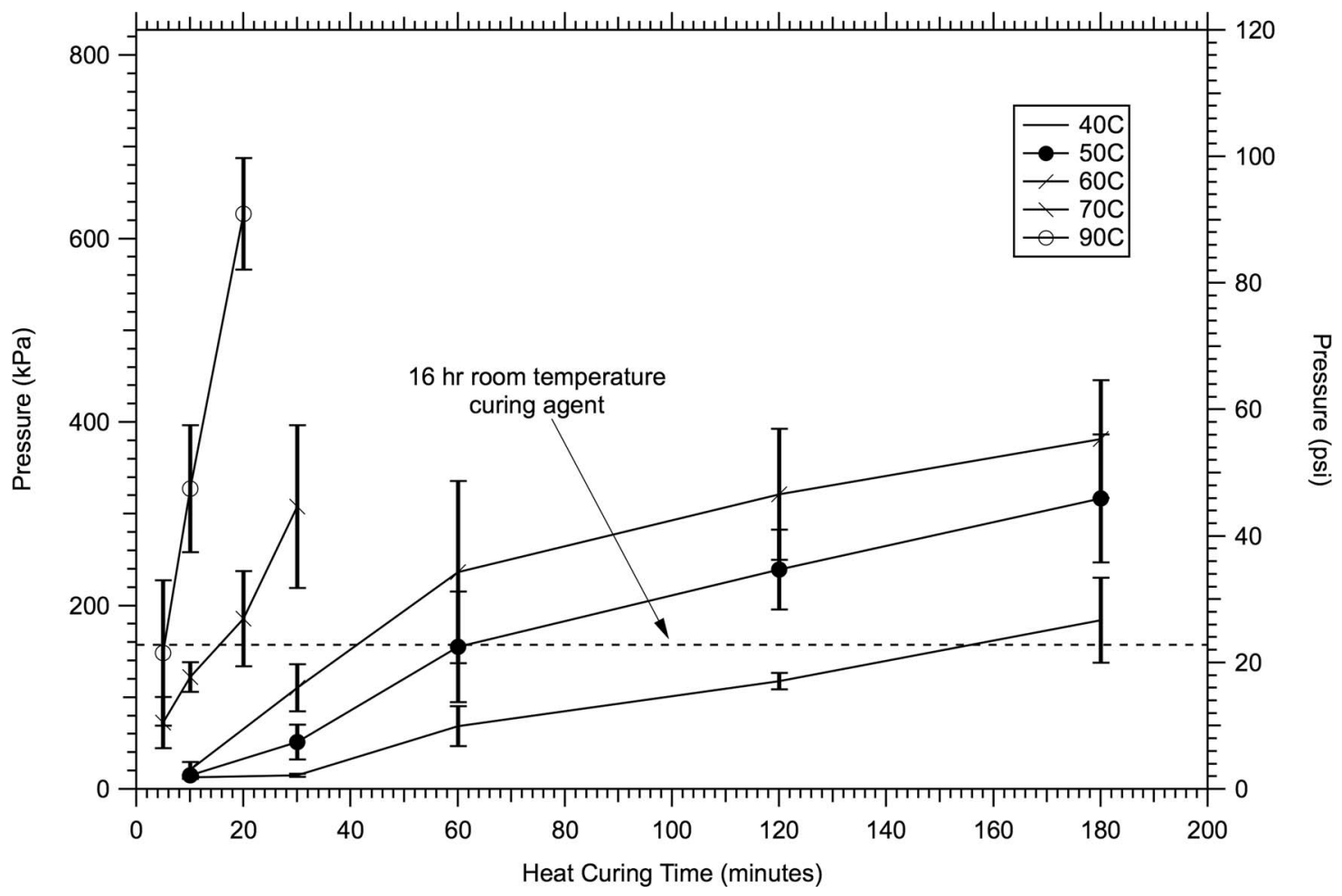

Fig. 6 Same as Fig. 5 except for Sylgard 182.

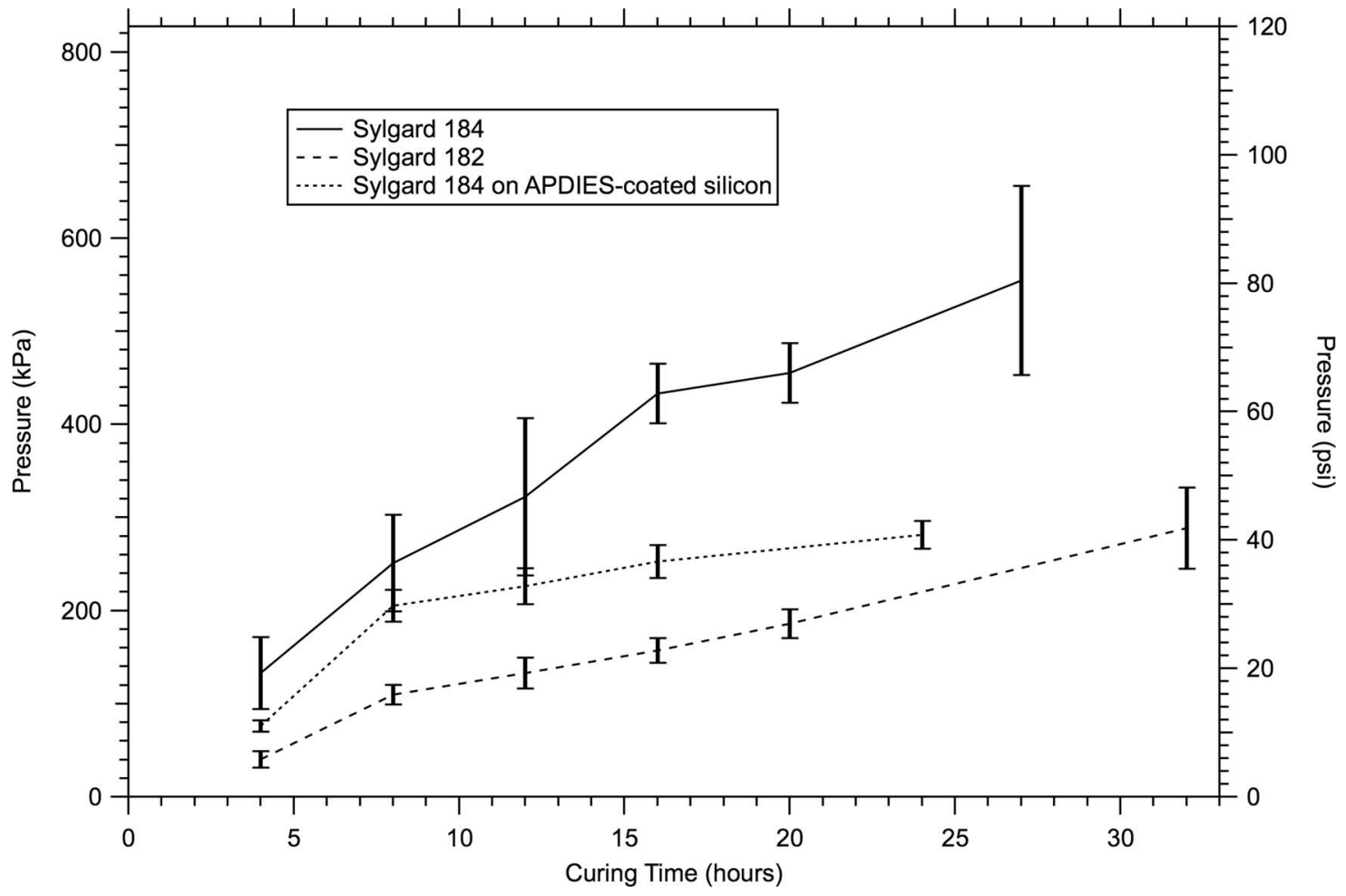

Fig. 7 Average measured burst pressure as a function of room temperature bonding time. 
Tsai et al.: Bonding of polydimethylsiloxane microfluidics to silicon-based sensors

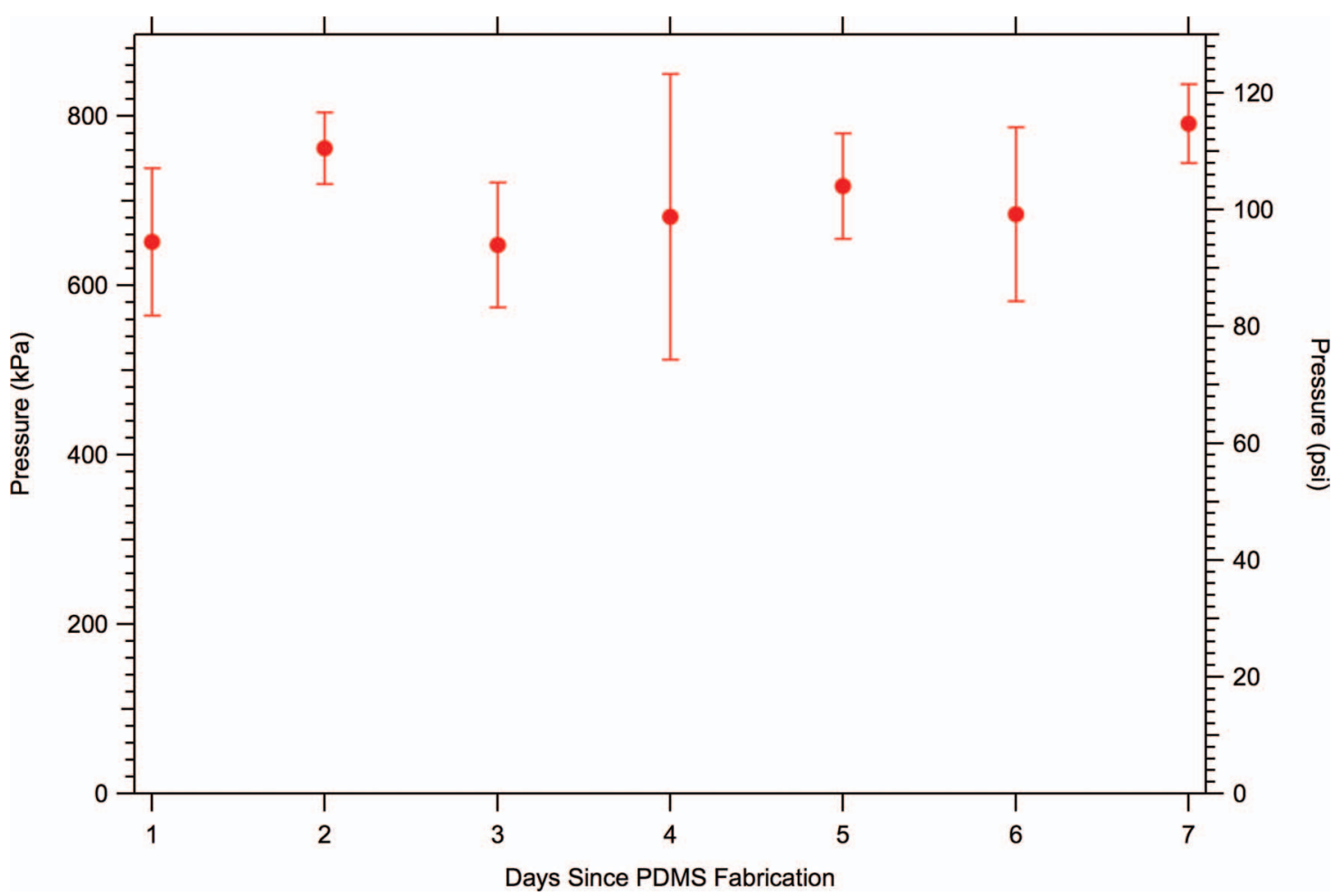

Fig. 8 Average measured burst pressure as a function of number of days since PDMS fabrication. All samples are bonded at $60^{\circ} \mathrm{C}$ for 60 min.

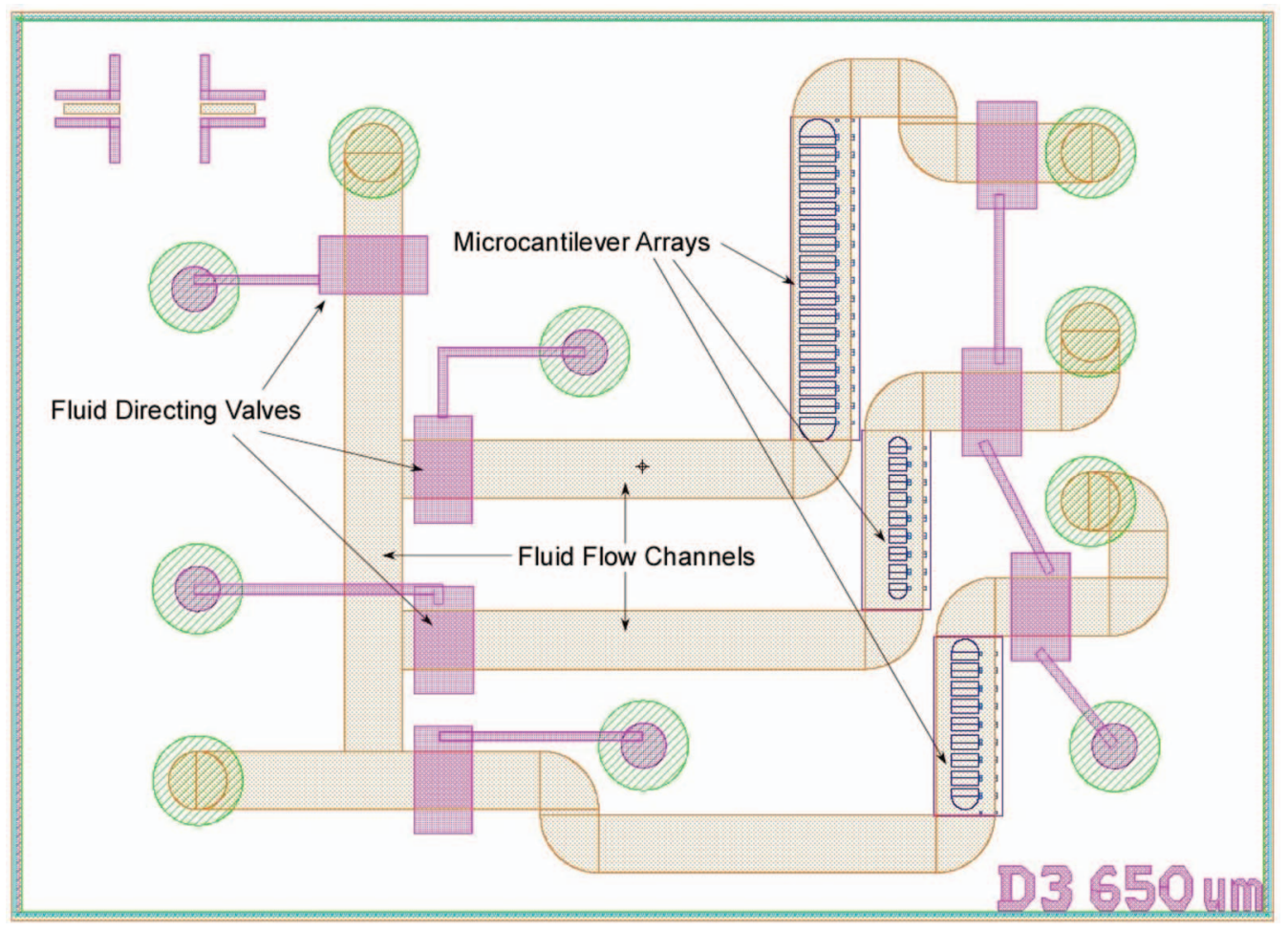

Fig. 9 Design layout of assembled PDMS-silicon sensor device. 


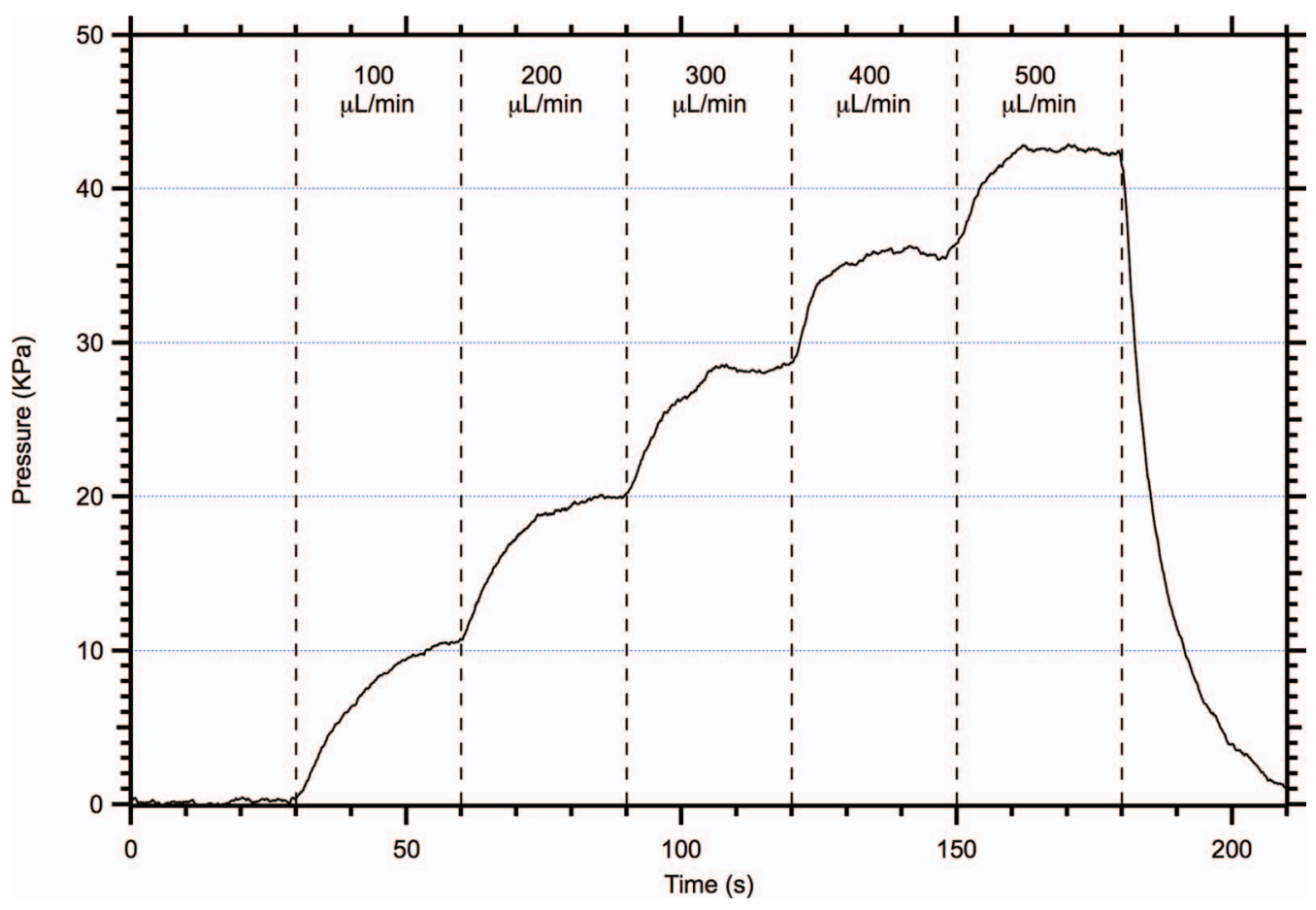

Fig. 10 Pressure in flow channel of sensing device as a function of time for increasing volumetric flow rates from 0 to $500 \mu \mathrm{lmin}{ }^{-1}$. Labels and dashed vertical lines indicate time intervals during which the indicated flow rates are generated with a syringe pump.

the average burst pressure does not decrease significantly until approximately 3 weeks after PDMS fabrication.

We also measured the pressure in an actual biosensing silicon-on-insulator device with integrated multilayered Sylgard 184 PDMS microfluidics and immobilized proteins on arrays of microcantilevers ${ }^{4-6}$ in order to qualitatively understand the applicability of the burst pressures attained by using an intermediate adhesive. An image of the design layout of the assembled sensor is shown in Fig. 9. It is assembled using the same procedure as in Fig. 3 with curing agent as the adhesive which is allowed to cure for approximately $16 \mathrm{~h}$ at room temperature. Different from the test devices, this sample has an input and outputs for desired fluids with channels $60 \mu \mathrm{m}$ high $\times 600 \mu \mathrm{m}$ wide. Since the adhesive layer is so thin $(\sim 2 \mu \mathrm{m})$ compared to the channel height, we find there is no issue with the adhesive layer affecting the fluid channels (e.g., spreading into the channels).

Pressure is induced at the PDMS-silicon interface as a function of the flow rate through the channel. The flow is into the fluid input in the lower right with valves arranged to direct the flow through one of the microcantilever array channels. Figure 10 shows the pressure as a function of time for flow rates up to $500 \mu \mathrm{l} \mathrm{min}{ }^{-1}$ using the test setup of Fig. 1. Note that this flow rate is significantly higher than the maximum flow rate we typically use in an experiment, which is $100 \mu \mathrm{min}^{-1}$ (and this is only used when we queue up a new fluid in the left vertical flow channel in preparation to introduce it to one of the microcantilever channels). During actual sensing runs flow rates of 2 to $5 \mu 1 \mathrm{~min}^{-1}$ are typically used.

Extensive experience with actual microfluidic-onmicrocantilever samples $(>30)$ shows that room temperature overnight $(\sim 16 \mathrm{~h})$ bond strength with Sylgard 184 on aminosilane-coated silicon is entirely adequate for the flow rates and pressures at which we typically operate. However, in our limited experience ( $\sim 5$ samples), use of Sylgard 182 results in too frequent PDMS delamination (nearly 50\% of the time), and direct bonding of PDMS with no intermediate adhesive layer or treatment always results in delamination.

\section{Summary}

We have evaluated PDMS curing agents for Sylgard 184 and 182 as intermediate adhesive layers as a function of curing time and temperature, and compared to NOA 75 UV curable epoxy. We find that Sylgard 184 is superior to Sylgard 182 in terms of bond strength for the same curing conditions. It also yields higher burst pressures than NOA 75 for nearly all investigated curing conditions in which the curing temperature is above room temperature. Moreover, we find that room temperature curing of Sylgard 184 for $16 \mathrm{~h}$ or longer gives average burst pressures of $433 \mathrm{kPa}$ or more.

\section{Acknowledgments}

This work was supported in part by NSF Grant Nos. ECS0602261 and IIS-0641973, and DARPA Grant No. 6600104-8933.

\section{References}

1. P. E. Sheehan and L. J. Whitman, "Detection limits for nanoscale biosensors," Nano Lett. 5(4), 803-807 (2005).

2. T. M. Squires, R. J. Messinger, and S. R. Manalis, "Making it stick: convection, reaction and diffusion in surface-based biosensors," Nat. Biotechnol. 26(4), 417-426 (2008).

3. J. W. Noh, R. Anderson, S. Kim, J. Cardenas, and G. P. Nordin, "Inplane photonic transduction of silicon-on-insulator microcantilevers," Opt. Express 16(16), 12114-12123 (2008). 
4. W. Hu, R. Anderson, Y. Qian, J. Song, J. W. Noh, S. Kim, and G. P. Nordin, "Demonstration of microcantilever array with simultaneous readout using in-plane photonic transduction method," Rev. Sci. Instrum. 80, 085101 (2009).

5. J. W. Noh, R. Anderson, S. Kim, W. Hu, and G. P. Nordin, "Sensitivity enhancement of differential splitter-based transduction for photonic microcantilever arrays," Nanotechnology 21, 155501 (2010).

6. R. R. Anderson, W. Hu, J. W. Noh, W. C. Dahlquist, S. J. Ness, T. M Gustafson, D. C. Richards, S. Kim, B. A. Mazzeo, A. T. Woolley, and G. P. Nordin, "Transient deflection response in microcantilever array integrated with polydimethylsiloxane (PDMS) microfluidics," Lab Chip 11(12), 2088-2096 (2011).

7. C.-C. Lee, G. Sui, A. Elizarov, C. J. Shu, Y.-S. Shin, A. N. Dooley, J. Huang, A. Daridon, P. Wyatt, D. Stout, H. C. Kolb, O. N. Witte, N. Satyamurthy, J. R. Heath, M. E. Phelps, S. R. Quake, and H.-R. Tseng, "Multistep synthesis of a radiolabeled imaging probe using integrated microfluidics," Science 310(5755), 1793-1796 (2005).

8. B. Samel, V. Nock, A. Russom, P. Griss, and G. Stemme, "A disposable lab-on-a-chip platform with embedded fluid actuators for active nanoliter liquid handling," Biomed Microdevices, 9, 61-67 (2007).

9. J. C. McDonald, D. C. Duffy, J. R. Anderson, D. T. Chiu, H. Wu, O. J. Schueller, and G. M. Whitesides, "Fabrication of microflu- idic systems in poly(dimethylsiloxane)," Electrophoresis 21, 27-40 (2000).

10. D. C. Duffy, J. C. McDonald, O. J. A. Schueller, and G. M. Whitesides, "Rapid prototyping of microfluidic systems in poly(dimethylsiloxane)," Anal. Chem. 70, 4974-4984 (1998).

11. S. Bhattacharya, A. Datta, J. M. Berg, and S. Gangopadhyay, "Studies on surface wettability of poly(dimethyl) siloxane (pdms) and glass under oxygen-plasma treatment and correlation with bond strength," J. Microelectromech. Syst. 14, 590-597 (2005).

12. L. Kersey, V. Ebacher, V. Bazargan, R. Wang, and B. Stoeber, "The effect of adhesion promoter on the adhesion of PDMS to different substrate materials," Lab Chip 9, 1002-1004 (2009).

13. S. Satyanarayana, R. N. Karnik, and A. Majumdar, "Stamp-and-stick room-temperature bonding technique for microdevices," J. Microelectromech. Syst. 14(2), 392-399 (2005).

14. W. P. Maszara, G. Goetz, A. Caviglia, and J. B. McKitterick, "Bonding of silicon wafers for silicon-on-insulator," J.Appl. Phys. 64(1D), 49434950 (1988).

Biographies and photographs of the authors are not available. 